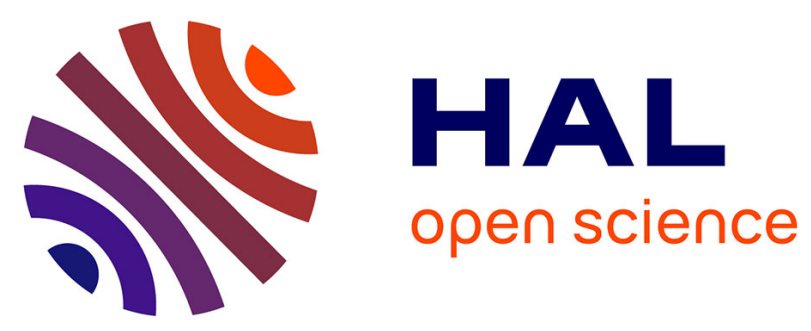

\title{
Tala Sound, un Projet Interdisciplinaire Innovant en Design Social pour la Santé
}

\author{
Luc Perera, Pierre Jouvelot
}

\section{To cite this version:}

Luc Perera, Pierre Jouvelot. Tala Sound, un Projet Interdisciplinaire Innovant en Design Social pour la Santé. Innovations - Revue d'économie et de management de l'innovation, A paraitre. hal-03137520

HAL Id: hal-03137520

https: / hal-mines-paristech.archives-ouvertes.fr/hal-03137520

Submitted on 10 Feb 2021

HAL is a multi-disciplinary open access archive for the deposit and dissemination of scientific research documents, whether they are published or not. The documents may come from teaching and research institutions in France or abroad, or from public or private research centers.
L'archive ouverte pluridisciplinaire HAL, est destinée au dépôt et à la diffusion de documents scientifiques de niveau recherche, publiés ou non, émanant des établissements d'enseignement et de recherche français ou étrangers, des laboratoires publics ou privés. 


\title{
Tala Sound, un Projet Interdisciplinaire Innovant en Design Social pour la Santé
}

\author{
Luc PERERA \\ Groupe Symbiose, Ecole national supérieur des Arts Décoratifs, Université PSL, Paris \\ luc.perera@ensad.fr \\ Pierre JOUVELOT \\ MINES ParisTech, Université PSL, Paris, \\ pierre.jouvelot@mines-paristech.fr
}

\begin{abstract}
RESUME
La musicothérapie s'avère être une des pratiques les plus utiles pour pallier, en partie, les conséquences néfastes des démences liées à l'âge. Forts de ce constat, nous avons initié le développement, pour des patients Alzheimer, d'un système sonore à visée médicale conçu autour de rythmes indiens carnatiques, ou talas. Nous présentons ici une analyse du déroulé temporel de cette "recherche par le design", en cours, afin d'en exhiber les retombées méthodologiques, en particulier en termes de design sonore interdisciplinaire et social. Notre conclusion est qu'une nouvelle approche méthodologique de la recherche par le design sonore pour la santé pourrait se fonder sur la création de liens sociaux et interdisciplinaires forts entre tous les intervenants des domaines de recherche impliqués. La valeur globale de ce type de projet semble en effet profiter grandement des principales retombées qu'une approche de design interdisciplinaire et social induit, de par l'apparition de synergies entre des disciplines non corrélées comme, dans le cas d'étude Tala Sound évoqué ici, la santé, l'anthropologie, le social, l'enseignement et l'ingénierie.
\end{abstract}

Mots-clés : Design sonore, Alzheimer, Méthodologie, Interdisciplinarité, Social.

Code JEL : O330

\section{Tala Sound, an Innovative Interdisciplinary Project in Social Design for Health}

\begin{abstract}
Music therapy is one of the most useful approaches to address, in part, the dire consequences of agelinked dementia. Building upon such a knowledge, we initiated the development, for Alzheimer patients, of a medical sound system designed around Carnatic Indian rhythms, or talas. We present here an analysis of the time course of a "research by design", in progress, in order to exhibit its methodological consequences, in particular regarding interdisciplinarity and social factors. Our conclusion is that a new methodological approach to sound-design-based research for health could be built on the creation of strong social and interdisciplinary links among all the stakeholders in the research fields involved. The total value of this kind of projects appears indeed greatly enhanced by the main outcomes that an interdisciplinary and social design approach yields, leading to synergies between apriori uncorrelated disciplines such as, in the case of the Tala Sound case mentioned here, health, anthropology, social issues, education and engineering.
\end{abstract}

Keywords: Sound Design, Alzheimer, Methodology, Interdisciplinarity, Social.

JEL code: O330 


\section{Introduction}

Les maladies neurodégénératives, qui touchent particulièrement la population des plus de 60 ans, représentent un problème de santé publique majeur. Ainsi, en 2014, environ $900000^{1}$ personnes souffraient de la maladie d'Alzheimer en France ; elles devraient être 1,3 million en 2020. Si des approches médicamenteuses ont été initialement tentées pour pallier ces troubles cognitifs, elles ne peuvent, jusqu'à présent, être considérées comme satisfaisantes. C'est dans ce contexte médical et, plus largement, politique, social voire pédagogique de mise en avant de solutions non médicamenteuses pour les démences que notre projet d'innovation s'inscrit. Et c'est à partir de ce constat que nous avons été amenés à décliner une méthodologie à même de mobiliser des partenaires de différentes disciplines autour d'un objectif commun de recherche par le design qu'est la création d'un nouvel objet visant à utiliser la musique comme outil d'accompagnement de ces patients : tel est le but du projet Tala Sound.

Le design sonore médical fait partie des disciplines émergentes. Il est, par nature, interdisciplinaire, si l'on en croit la définition proposée par Hamel, à savoir que l'interdisciplinarité est " [1'] utilisation combinée de quelques disciplines, combinaison entraînant des transformations réciproques dans chacune d'elles. » (Hamel, 1995, p. 61), car il allie à la fois le monde des arts appliqués, la musique et la santé, tout ceci ayant vocation, dans notre projet, à s'articuler autour d'un dispositif sensoriel comme l'est également le snoezelen (Fanny, 2000). La mise en place de ce type de projet demande, naturellement, une connaissance de l'existant, la définition d'une stratégie et d'une méthodologie de recherche interdisciplinaires. Ces problématiques méthodologiques concernent d'ailleurs aussi bien l'organisation de la recherche menée que la stratégie de celle-ci.

Dans le projet Tala Sound, l'innovation visée a été la conception d'un objet prétexte, car « il ne s'agissait pas de rebasculer dans un monde social où les objets ne comptent plus que comme prétextes ou symboles, mais de retrouver un monde mêlé [...], des pratiques, des entraînements, des associations de comportements, tout aussi incapables de déterminer leur objet que celui-ci de déterminer son usage. \ (Dubuisson, 1996, p. 14). Ainsi dans notre vision, cet objet musical aura vocation à créer du lien social entre les personnes et les disciplines tout en influant sur le processus créatif même, comme le dit Bontems : "In principle, innovation bears no relation with scientific research, but is often based on the adoption of new objects or new uses of existing objects. » (Bontems, 2014, p. 45). Ces éléments de réflexion nous ont amenés à envisager une question centrale encore plus large que celle du "simple" design d'un objet sonore à finalité de soin : Un design interdisciplinaire répondrait-il donc, ainsi, in fine, à un problème sociologique?

Pour aborder cette question et proposer des éléments de réponse méthodologique, nous avons effectué une analyse du déroulé temporel d'une recherche de design sonore en cours, à savoir la conception de la Tala Box, un dispositif sonore à visée médicale conçu autour des rythmes, ou talas, indiens carnatiques pour les patients atteints de la maladie d'Alzheimer. Celle-ci va nous conduire à suggérer qu'une approche méthodologique de la recherche par le design

\footnotetext{
${ }^{1}$ https://alzheimer-recherche.org/la-maladie-alzheimer/quest-maladie-dalzheimer/definition-et-chiffres/
} 
pourrait se fonder sur la création de liens sociaux forts entre tous les intervenants des domaines de recherche impliqués.

Pour décrire ici ce déroulement temporel méthodologique, nous avons, dans la section 1, positionné le contexte en identifiant les besoins dans le domaine de la gériatrie et du design sonore, et nous avons identifié la faisabilité et l'utilité des rythmes carnatiques dans un hôpital public. La section 2 décrit l'approche prise pour, autant que possible, valider nos hypothèses médicale et musicale. La section 3 illustre ces réflexions à travers le design du projet social Tala Sound, concrétisé par l'objet Tala Box. Nous discutons les principaux traits saillants du déroulé de ce projet dans la section 4 , avant de conclure.

\section{Positionnement et Contexte}

\section{Identification des besoins d'usage}

La proposition méthodologique qui va être faite ici est fortement liée à l'historique original de notre recherche sur Tala Sound, dont nous avons abordé les prémices dès 2015. Le choix ethnomusicologique du tala, une notion clé de la musique indienne concernant la structure rythmique des œuvres, comme fondement de notre recherche n'est pas dû au hasard. Tout d'abord, un des auteurs a fréquemment voyagé entre la France et l'Inde du Sud pendant plus de dix ans, étant de fait baigné dans la matière sonore de ces deux pays dès l'enfance. Ses compétences en pratique et composition musicales ont ainsi constitué le terreau de Tala Sound, alors que sa profession d'enseignant dans le domaine du design lui a permis de suivre quotidiennement une méthodologie largement pluridisciplinaire. La mise en contact, liée au parcours personnel de cet auteur, avec le docteur Anne-Laure Vétillard, du service de gériatrie de l'hôpital Paul Brousse à Villejuif (Val-de-Marne, France), a constitué le second facteur déclencheur. L'idée de chercher à lier design d'objet, rythmes indiens et prise en compte des troubles liés à l'âge a alors germé. On voit ici que le processus même de recherche contient dès l'origine des aspects sociaux clés, aspects dont nous aurons l'occasion de redécouvrir l'importance par la suite.

Lors d'une visite, le Dr Vétillard nous a conduits à découvrir son service de gériatrie, sans que nous ayons de contact direct avec les patients. Cette approche de découverte nous a permis d'identifier le travail des gériatres (Lauquin, Minvieille, 2015). Dans un second temps, nous avons participé dans le même lieu à une journée de « kermesse » durant laquelle les patients atteints de démence allaient à l'extérieur et «participaient » à des activités avec le personnel soignant et les membres des familles. A des fins de comparaison, nous avons établi un contact avec l'établissement d'hébergement pour personnes âgées dépendantes (EHPAD) «Denis Forestier » de la MGEN, qui se situe à La Verrière (Yvelines, France), via le Dr Montaner, qui y est médecin coordinateur. Nous avons visité deux bâtiments dès notre premier entretien : l'un pour personnes atteintes de démence faible et l'autre dédié aux cas sévères, la sévérité étant évaluée via le score MMS (Mini Mental State) (Crum et al., 1993). Cette phase d'observation nous a permis d'identifier le rôle de chacun et l'attribution des diverses tâches.

En se fondant sur les critères classiques du domaine du design, cette première démarche peut être qualifiée d'identification des besoins, comme cela se retrouve dans le design de nouveaux produits que pourrait initier un service marketing : "L'équipe de designers doit comprendre comment fonctionnent ces mécanismes internes et externes [des besoins] pour pouvoir ajouter de la valeur et créer un avantage compétitif, tout en soutenant la viabilité de l'entreprise. » 
(Best, 2009 p. 14). Nous avons aussi profité de ces premiers échanges pour obtenir des informations primordiales concernant la motivation, forte, du personnel soignant, la disponibilité d'espaces pour effectuer des tests et la disponibilité des patients. C'est alors seulement après coup que nous avons commencé à entrevoir la pertinence d'une recherche liant musique indienne et gériatrie. "Le sens dans l'action vient la plupart du temps "après coup". Il n'est donc pas immédiat, mais attribué a posteriori par une interprétation. " (Blais, Martineau, 2006, p. 3).

Pour mieux cerner cette problématique en cours d'élaboration, nous avons alors choisi de suivre les préceptes de l'analyse de l'existant, telle qu'elle est pratiquée en pédagogie des arts appliqués. Nous avons donc récolté divers textes de la littérature dans les domaines a priori pertinents, comme les sciences (neurologie et maladie d'Alzheimer ou des éléments de référence sur objet et design sonore, "le terme objet pouvant être pris au sens large d'objet tangible (produit manufacturé), d'objet numérique (interface homme-machine) ou d'objet spatial (environnement). » (Misdariis, 2018, p. 11), notions qui sont souvent associées dans les domaines du marketing (Bobrie, 2017, p. 96) ou de la communication marchande. Nous avons pu noter, de manière intéressante, qu'aucun article scientifique sur le design sonore médical ne semble exister. Cependant, nous avons pu trouver quelques projets d'étudiants de l'école ENSCI dont la forme était intéressante ${ }^{2}$. En particulier, on notera l'existence de quelques tentatives de design d'objet telles que l'horloge aide-mémoire, de Pierre Charrié, pour patients atteints d'Alzheimer (Bouillon et al., 2009), ou, pour des patients souffrant de troubles en neuropsychiatrie, Music Care ${ }^{\varpi}$, dont le dispositif a été testé en milieu médicalisé. Nous avons, par contre, trouvé de nombreuses études sur le bruit, comme Chelkoff (1993) ou Leroux et Bardyn (2003), ou le confort acoustique dans les hôpitaux, mais il convient de pas confondre ces aspects avec ce qui relève du design sonore médical. Pour finir, en ce qui concerne les liens entre milieux médical et sonore, quelques études existent sur les alarmes en néonatalogie (ARS, 2018) ou les stimulations sonores (Kuhn, 2012), ainsi que des études sur la qualité sonore d'appareils vocaux portatifs réalisés pour des patients ayant subi une laryngectomie (Hueber, 2009).

\section{Analyse de l'existant}

A partir des données issues de nos observations et de l'analyse préliminaire de la littérature, nous avons élaboré une carte mentale (voir la MindMap de la figure 1), qui nous a conduit à mettre en évidence deux grands groupes de concepts mobilisables : la musique et le monde médical. Dans le premier groupe, on distingue trois pôles : le son, la musique indienne et la méditation, qui lui est liée culturellement. Dans le second groupe, on retrouve ce qui concerne le patient, le milieu médical et les dispositifs contemporains. Si cette cartographie permet une lecture simple de l'idée principale de notre problématique, elle n'en dévoile pas tous les concepts de recherche sous-jacents. C'est toutefois grâce à cette constatation-déduction que nous avons pu élaborer notre hypothèse de recherche, qui apparait maintenant clairement comme relevant du domaine de la musicothérapie, discipline qui lie santé et musique (ou son). La littérature en musicothérapie est foisonnante. D'un point de vue moderne, cette pratique existe depuis les années cinquante : elle a d'abord été appliquée aux blessés post-traumatiques de la Seconde guerre mondiale pour calmer leurs insomnies. Et c'est à partir de 1954 que Jacques Jost réussit à valider l'efficacité de ces séances à la faculté de médecine de Paris. Le $1^{\text {er }}$ congrès mondial sur ce sujet en France s'est tenu en 1974. En ce qui concerne la méthodologie, on peut distinguer dans cette discipline deux types de démarches

\footnotetext{
${ }^{2}$ http://symbiose.ensadlab.fr/design-sonore-et-sante-au-mans/
} 
thérapeutiques: l'une est active, liée à la participation du patient afin de le conduire à communiquer verbalement, tandis que l'autre est réceptive et est fondée sur l'écoute d'extraits sonores. Dans les deux cas, le programme est typiquement réalisé suite à un entretien préalable, ainsi qu'un test de réceptivité musicale. Il est fragmenté en trois parties : apaisement, détente et relaxation, selon Jacques Jost, Edith Lecourt et Jaqueline Verdeau-Pailles (Vrait, 2018).

La musicothérapie agit de manière manifeste, via des canaux sensoriels, cognitifs et affectifs. Des recherches furent menées en neurologie par scanner pour en évaluer le mode d'action ; elles suggèrent que certaines zones spécifiques du cerveau réagissent à la musique (Moussard, 2012). On a constaté que les lobes dissociaient à la fois la musique et les paroles. Ainsi, un lobe cérébral peut réagir à une structure musicale (mélodies et rythmes) alors que les paroles non mélodiques sont traitées par l'autre lobe (Bigand et al., 2012). Des chercheurs ont, par ailleurs, mis en application la notion de «bande en U » (Guétin et al., 2009) pour évaluer l'impact de l'écoute musicale sur l'intensité de la douleur ressentie par les patients atteints d'Alzheimer en faisant varier rythmes, mélodies et fréquences. On la trouve aussi logiquement utilisée également comme technique de relaxation musicale personnalisée fondée sur l'hypnoanalgésie.

La recherche en musicothérapie est, de fait, internationale. Un des auteurs, d'origine indienne, s'est penché sur la question de la musicothérapie dans son pays d'origine. Dans cette partie du continent asiatique, on étudie les propriétés de la musique du Nord (hindoustani) dans les secteurs médicaux tels que l'addictologie, l'autisme ou l'obstétrique. Elle est également étudiée en lien avec l'état de stress de patients ou de sportifs, mais, à notre connaissance, aucun travail ne s'est jusqu'à présent penché sur le cas des patients atteints d'Alzheimer.

Comme dernier élément de cet état de l'art, il convient de citer une étude clinique descriptive très récente sur l'état de patients déments de type Alzheimer après une séance de musicothérapie, avant et après la prise de médicaments. L'étude semble mettre en évidence les effets bénéfiques de cette thérapie non médicamenteuse, qui a été réalisée dans le même hôpital que celui impliqué dans notre étude (Brahmi et al., 2019).

\section{Hypothèse de recherche}

La musicothérapie occidentale a donc largement prouvé son efficacité ; elle est approuvée par le milieu médical, l'Unité cognitive comportementale du CHU de Montpellier ayant ainsi classé, en 2017, la musicothérapie comme l'une des approches à privilégier dans les cas de troubles cognitifs, du fait de son influence positive et de son effet apaisant sur le comportement des patients. Notre positionnement de recherche va donc consister à essayer d'en étendre le champ d'influence sur la base des éléments de contexte précisés plus haut et en vertu du fait que la musique indienne est considérée, culturellement, comme possédant des propriétés apaisantes en utilisant, par exemple, les chants cycliques de nemboutsou (Larroque, 2013, p. 755).

Nous avons également tenu compte lors de la définition de notre approche de divers articles et ouvrages plus spécifiquement consacrés aux patients atteints de démence ainsi que de ceux abordant des dispositifs et axes de recherche non médicamenteux contre la démence. Cette étude bibliographique préliminaire nous a fait découvrir deux points-clés nous encourageant à affiner notre projet: (1) nous n'avons pas trouvé de publication en Inde liant musique traditionnelle et démence de type Alzheimer, et (2) les relations entre musiques extraeuropéennes et santé en Europe ne paraissent pas avoir été étudiées en profondeur ${ }^{3}$.

\footnotetext{
${ }^{3}$ Selon le Pr Edith Lecourt (communication privée, juin 2016)
} 
Notre recherche va donc consister à déterminer dans quelle mesure l'écoute de musiques extraeuropéennes d'origine indienne, médiée par un dispositif sonore adéquat, peut avoir un impact positif dans le traitement des démences, et ce dans une population a priori peu familière de ces sons. C'est à partir du moment où cette hypothèse de recherche a été clairement spécifiée qu'il nous a été possible d'envisager la mise en place d'une stratégie dédiée à la définition précise et à la validation de ce projet.

Figure 1 - MindMap du projet Tala Sound de design sonore médical

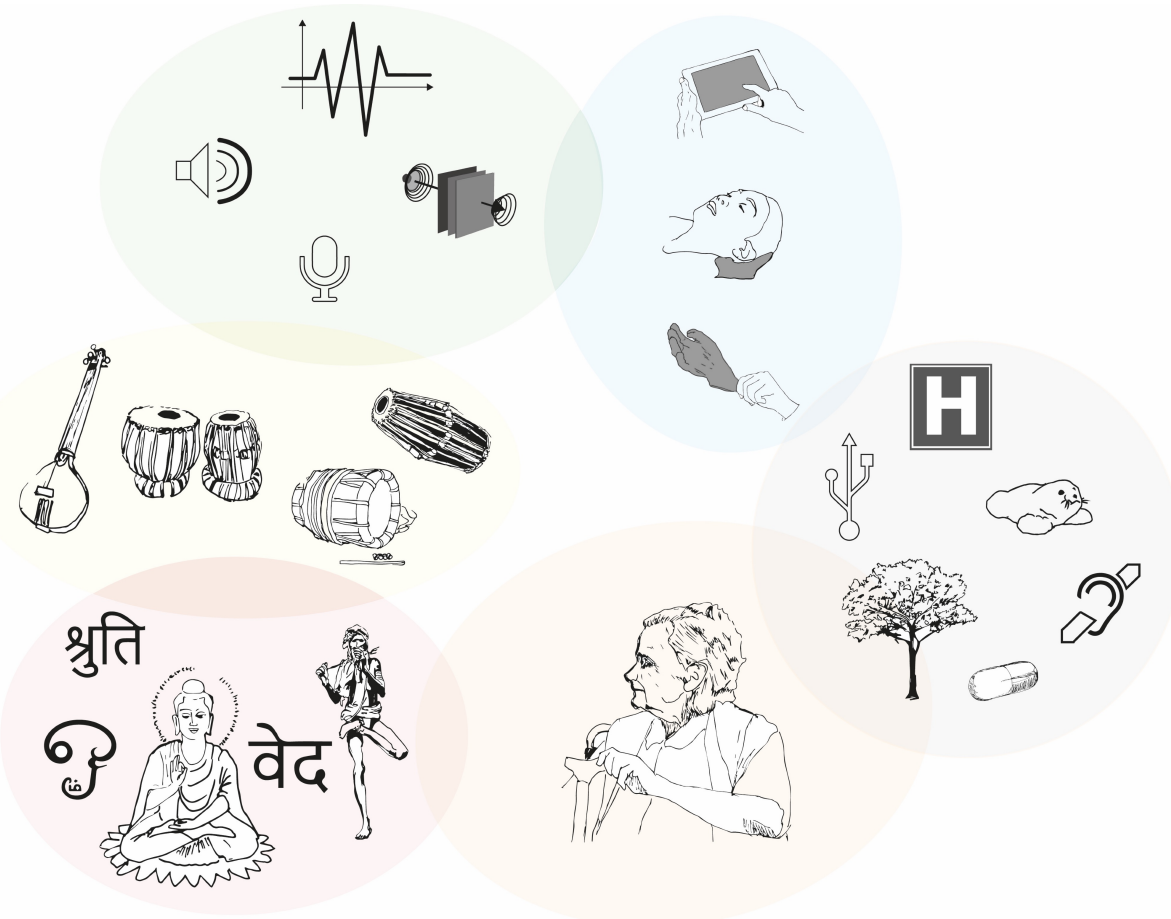

\section{Recherche et Disciplines Médicale et Musicale}

\section{Acceptabilité}

L'objectif de validation (ou non) de notre hypothèse impose d'aborder ce projet selon les principes méthodologiques suivis en recherche médicale, qui sont, dans ce domaine, très codifiés. Un des guides de référence est Méthodologie de la recherche dans le domaine de la santé, Guide de formation aux méthodes de la recherche scientifique, publié par l'Organisation mondiale de la santé en 2003. Selon cet ouvrage, la recherche liée à la médecine reste largement empirique, visant un certain nombre de populations bien définies. Souvent, les médecins procèdent de manière inductive, partant de faits pathologiques qu'ils essayent d'éliminer en cherchant des solutions validées expérimentalement.

En France, pour tout ce qui concerne la recherche, les hôpitaux publics et privés suivent un lourd protocole médical de recherche validé par les Comités de protection des personnes (CPP). Pour mettre en place ce protocole, il faut se fonder sur la loi Jardé ou le règlement associé de l'Union européenne. Toutefois, depuis le mois de mai 2017, les recherches en sciences 
humaines et sociales, du type de celles habituellement liées au domaine du design, ne sont plus considérées comme des recherches médicales ; le décret JOFR $\mathrm{n}^{\circ}$ 2017-884 du 10 mai 2017 stipule que «II.- $1^{\circ}$ Ne sont pas des recherches impliquant la personne humaine au sens du présent titre les recherches qui, bien qu'organisées et pratiquées sur des personnes saines ou malades, n'ont pas pour finalités celles mentionnées au I, et qui visent [...] :c) A effectuer toute autre enquête de satisfaction auprès des patients. » Tant que les phases d'évaluation sont réalisées par enquête de satisfaction, les études associées n'auront pas le statut de « recherche » au sens médical du terme.

Comme un de nos objectifs est d'allier, dans un temps raisonnable, projet de design et mise en évidence aussi quantitative que possible de son impact sur le public visé, il nous faudra donc restreindre notre méthodologie à ce type de technique d'évaluation pour nos observations empiriques. Si les données obtenues grâce à ces questionnaires sont en quantité suffisante, on pourra envisager d'effectuer quelques analyses statistiques, toutefois forcément limitées. De plus, dans le cas de patients encore peu atteints par la démence, nous envisageons d'effectuer puis d'analyser des enregistrements audionumériques voire vidéo, si nous obtenons leur accord pour ce faire. De cette manière, nous pouvons espérer obtenir des "verbatim" intéressants quant à la réception du projet Tala Sound.

Pour fournir des premiers éléments convaincants de la pertinence de notre approche, et ce afin de la tester en milieu médical, il fallait toutefois déjà expérimenter et vérifier de manière anecdotique notre hypothèse concernant l'effet potentiellement apaisant de la musique indienne auprès d'un auditoire peu familier de ce type de sonorités comparé à un public indien (Kalyani et al., 2011) (Hegde, 201). Certes, en Occident, le rapport entre le corps et la musique a souvent été traité. On y trouve des références dès l'Antiquité avec l'exemple de la République de Platon, lors d'une discussion entre Glaucon et Socrate au sujet de la gymnastique et de la musique : " [...] l'éducation musicale est souveraine parce que le rythme et l'harmonie ont au plus haut point le pouvoir de pénétrer dans l'âme et de la toucher fortement, apportant avec eux la grâce et la conférant, [...] » (Platon, 2016, 4011d). Il s'agissait là des prémices de ce qu'est l'expérience musicale. Mais, dans notre cas, l'utilisation de musiques extra-européennes pouvait être, ou non, un facteur contre-productif.

Pour vérifier ce point important d'acceptabilité, nous avons réalisé une recherche exploratoire avec un groupe limité de 43 personnes des deux sexes et âgées de 25 à 50 ans, lors d'un concert live, le soir, pendant une heure. Nous avons pu observer que les musiques hindoustani (du Nord de l'Inde) et carnatique (du Sud de l'Inde) jouées à cette occasion étaient considérées comme apaisantes par $90 \%$ des personnes interrogées. Forts de ce premier résultat encourageant, nous avons fait une seconde évaluation, mais, cette fois-ci, dans un centre d'accueil pour personnes âgées, un public plus représentatif de celui que nous visions. Le résultat fut, là également, concluant, de l'avis même des participants. Dans ce cas, toutefois, les conditions nous ont conduits à réduire la durée d'écoute - pour s'adapter au degré souvent limité d'attention des personnes âgées - et à nous concentrer sur la musique carnatique, plus centrée sur le rythme. Pour définir ce paramètre temporel, nous avons pour seule référence directe une étude portant sur une borne musicale ${ }^{4}$ effectuée au CHU de Toulouse, pour laquelle la durée approximative d'écoute ${ }^{5}$ était analysée. De manière plus anecdotique, selon une étude sur la pratique culturelle des Français de 1997 à 2008 (Donnat, 2009, p. 4), les personnes âgées ont toujours une

\footnotetext{
${ }^{4} \mathrm{https}$ //www.francetvinfo.fr/replay-radio/france-info-seniors/une-borne-musicale-qui-change-le-quotidien-desaines_1768005.html

${ }^{5} \mathrm{https}$ ://www.chu-Toulouse.fr/IMG/pdf/MELO_Doc_Site.pdf
} 
appétence marquée pour l'écoute, en l'occurrence radiophonique, cette activité étant pratiquée par 58\% de la population cible en 1997 et $62 \%$, en 2008 ; certes, il convient de préciser que nous n'avons pas d'idée claire de leur «concentration » pendant ces phases d'écoute. Il faudra donc évaluer plus tard ce temps de concentration pendant l'écoute des sonorités carnatiques, quand nous la mettrons en place dans les hôpitaux.

Au vu de ces premiers sondages expérimentaux, il s'avérait de fait possible d'envisager que la musique indienne soit reconnue comme apaisante, non seulement lors des séances de méditation (yoga) où on la trouve habituellement cantonnée, mais aussi dans des environnements éloignés de ce type de problématique. Ceci nous a confortés dans l'idée de mettre en œuvre ce genre d'activités (écoute et phase d'observation) dans un hôpital ou un EHPAD. Notre objectif consistera donc à étendre l'usage des sonorités indiennes en fonction des réactions des auditeurs, en suivant le précepte selon lequel «L'histoire des récentes technologies à succès montre que les usagers sont aussi souvent des acteurs essentiels de l'innovation » (Ambrosi et al., 2005, p. 85) et en cherchant ainsi à court-circuiter les méthodologies classiques de design industriel : «[ ...] l'histoire récente de l'innovation dans les secteurs de l'informatique et des télécoms montre que cette intervention en amont des analyses d'usages est souvent courtcircuitée par des innovations qui trouvent leur origine hors des laboratoires, dans des lieux et des espaces sociaux qui échappent largement à l'attention des industriels. » (Cardon, 2006, p. 5) voire à concevoir une extension (Akrich, 1998) de l'usage d'un dispositif selon les résultats.

\section{Composition}

Dans la littérature que nous avons citée à propos de la musicothérapie, les chercheurs ont clairement identifié que les rythmes sonores impactaient le cortex moteur (Morillon, Baillet, 2017) et que l'enregistrement des «séquences rythmiques » se développait même avant l'enfance. Une étude a ainsi été réalisée avec des bébés singes, en analysant les effets de tapotements synchronisés selon des intervalles rythmiques (Crowe et al., 2014); les chercheurs ont pu montrer que les périodes temporelles s'enregistraient effectivement dans la mémoire. On sait par ailleurs que les souvenirs musicaux, et donc rythmiques, sont particulièrement bien préservés chez les patients âgés souffrant de démence cognitive. Notre proposition musicothérapeutique s'appuiera donc sur l'écoute de séquences essentiellement rythmiques (talas carnatiques) contenant des sons percussifs, des silences et des jeux mélodiques, successivement moderato et pianissimo pour en accroitre l'intérêt. En s'appuyant sur le fait que les rythmes participent à la sensibilité de la perception auditive même chez les patients atteints de démence, tout comme ils le font sur des personnes saines, nous pouvons émettre l'hypothèse que ces rythmes dédiés vont interférer de manière positive avec les processus cognitifs apparentés présents dans le cerveau, comme cela a été montré par l'étude de Fujioka (Fujioka et al., 2012, p. 1797).

En pratique, pour mettre en place l'aspect proprement musical de ce processus de recherche en design sonore, nous nous sommes fondés sur une méthodologie classique de composition musicale. Benjamin Martin, chercheur en informatique musicale, évoquant les spécificités de la perception rythmique, "[...] souligne que notre cerveau effectue naturellement un découpage des événements selon des groupes en se basant sur de nombreux critères liés aux éléments percussifs, à la dynamique, à la hauteur des notes ou encore au timbre du son.» (Martin, 2012, p. 18). Nous avons décidé de travailler sur une structure rythmique traditionnelle nommée Din Tala, que nous avons restructurée dans un tempo "classique » à 80 BPM (beat per minute) afin de maintenir l'illusion d'un écoulement du temps plus proche des canons « occidentaux ». Nous n'avons pas effectué ici de détournement majeur, car, dans certaines 
compositions de musique classique indienne, des artistes utilisent d'ailleurs ce système occidental pour créer des phrases musicales A/B/A (couplet/refrain/couplet) : "Dans leur musique se mêlent des formules spécifiques des rythmes carnatiques et, dans une moindre mesure, les séquences rythmiques hindoustani (notamment les tihai et des tekha avec leurs chal spécifiques), des grooves en 4/4, avec des arrangements mélodiques combinant bandish et saval-javab indiens, des phrasés jazz et des ponctuations spécifiquement flamencas. » (Bourgeau, 2008, p. 177). Nous avons introduit plusieurs structures rythmiques, que nous avons sélectionnées puis échantillonnées pour créer cette illusion de phrases $\mathrm{A} / \mathrm{B} / \mathrm{A}$.

Pour accentuer les effets graves du bay $a^{6}$, nous avons superposé ses interventions avec celles du tanpura ${ }^{7}$ et fait jouer la basse avec des sawara $^{8}$ dans un mode (thaats) que l'on nomme Asavari (mode dans lequel les "notes » GA et DHA sont 1/2 ton au-dessus de celle du ré majeur qui nous a servi de référence). Notons qu'il n'y a pas de râga (ou élément mélodique préconçu) dans ces compositions dédiées, mais seulement un jeu improvisé, ce qui permet une perception acoustique de la musique indienne par les auditeurs axée essentiellement sur les éléments rythmiques. L'objectif principal est clairement ici de faire réagir les auditeurs à la structure temporelle induite par le jeu sur tablas, en en accentuant d'ailleurs les sons graves. In fine, nous avons organisé des séances d'enregistrement avec des musiciens spécialistes de la musique indienne que nous avons choisis en fonction de leur compétence, afin de concrétiser ces idées sous forme sonore. Ces séances furent nombreuses et variées, car nous souhaitions expérimenter avec toutes les phases d'exécution rythmique envisageables à partir des structures existant dans les différents talas carnatiques.

Ces compositions musicales, prêtes au moment de l'écriture de cet article, ont commencé à être diffusées et commentées au cours de séances début 2019 auprès de patients de 1'hôpital Paul Brousse atteints de la maladie d'Alzheimer. Cette première phase d'expérimentation d'écoute simple va nous permettre par ailleurs d'évaluer, de manière anecdotique, dans quelle mesure cette écoute musicale permet éventuellement de créer du lien entre patients et soignants et, si oui, de quel(s) type(s), liant ainsi encore considérations médicales et sociales.

\section{Un Projet Pédagogique pour un Design Social}

\section{Conceptualisation d'un design par projet}

Le design par projet est un «processus unique qui consiste en un ensemble d'activités coordonnées et maîtrisées comportant des dates de début et de fin, entrepris dans le but d'atteindre un objectif conforme à des exigences spécifiques, incluant les contraintes de délais, de coûts [...] » (AFITEP, 2010, p. 211). Il est «méthodique » (Vial, 2015, p. 15), en ce qu'il se fonde sur une anticipation des différentes phases de recherche. C'est aussi là un des aspects clés du projet Tala Sound, qui a pris le parti, dès le début, d'aller au-delà de la simple écoute musicale et de concevoir un objet sonore et tangible innovant permettant, du moins l'espérons-

\footnotetext{
${ }^{6}$ Tabla : Instrument de percussion issu de l'Inde du Nord, comportant deux fûts distincts : le tabla proprement dit, qui fait $27 \mathrm{~cm}$ de haut et dont la caisse est en bois, la peau de chèvre produisant des sons aigus, et le baya, qui fait $26 \mathrm{~cm}$, est en cuivre et produit des sons graves.

${ }^{7}$ Tanpura : Instrument de musique indienne qui ressemble à un luth mais qui a seulement 4 ou 6 cordes et qui produit des sons continus, comme un bourdon.

${ }^{8}$ Séquences de notes de musique indienne.

${ }^{9}$ Nous pourrions faire un parallèle avec la gamme de Ré majeur.
} 
nous, de renforcer les liens patients-soignants (Côté et al., 2017, p. 56). Dès 2016, nous avons planifié les différentes étapes du processus.

L'importance de la médiation par objet dans les cas de démence est bien connue : voir, par exemple, le cas du phoque robotisé Paro (Moyle et al., 2013), qui conforte l'idée de notre hypothèse initiale. Notre but principal consiste donc à créer un objet médiateur (Gagnon, 1986) à visée médicale qui pourrait être considéré comme « innovant» par l'utilisation de sonorités non-européennes. Nous avons pris le parti de nous placer dans l'optique d'une conception sociale (Gagnon, Côté, 2016, p. 3) de l'usage par l'objet. Le patient, le personnel soignant et la famille (nous l'espérons) pourront communiquer ensemble, à l'image de ce qui est fait dans certaines recherches en art-thérapie dans le domaine pédiatrique, car la médiation a "un pouvoir de socialisation, à travers, par exemple, des activités de groupe, génératrice de la communication et du loisir » (Ferraro, 2013, p.178). Il est évident qu'il faudra naturellement mettre en place des phases de consentement entre les deux sujets que sont patient et famille ou tuteur (Thorez et al., 2009).

La réalisation pratique de la Tala Box nécessite toutefois naturellement la participation de spécialistes en ingénierie. Nous avons décidé de continuer à jouer sur l'interdisciplinarité de notre projet, voire sur les liens entre design, interdisciplinarité et ingénierie (Gentes, 2017) et l'accent mis sur la part humaine et sociale que nous pensons pouvoir loger au cœur de tout processus de design ; ce consensus de l'ensemble des acteurs (Debos et al., 2017 p.3) sousentend que nous envisageons là, nous l'avons vu, un processus dans lequel l'utilisateur patient, famille et personnel soignant auront un rôle actif dans la conception du produit (Guilloux, Le Bœuf 2017). De même, pour la réalisation, si nous aurions pu directement nous adresser à des ingénieurs professionnels, nous avons préféré intégrer à notre processus de recherche le lycée technique Gaspard Monge, à Savigny-sur-Orge (Essonne, France). En effet, nous avons estimé qu'une passerelle sociale plus large aurait l'avantage de décloisonner les pratiques entre l'enseignement supérieur et le secondaire, de servir de moteur d'orientation pour de futurs ingénieurs ou designers et d'être la source de processus créatifs non prévus tout en cadrant avec une forme de « cohésion sociale » (Beauvais et Jenson 2002).

Comme un des auteurs de l'étude est également enseignant dans le secondaire, son expérience nous a permis de travailler la notion d'apprentissage intergénérationnel et, plus précisément, celle des générations socio-historiques (Hummel, Hugentobler, 2007, p.79). Le principe est donc de créer du lien par la pédagogie et la didactique, comme l'a réalisé le réseau NIGEL en Suisse, qui intègre les enfants dans des projets intergénérationnels. L'objectif est de permettre à une génération d'inventer (Choain, 2014, p.134) en se fondant sur l'existant. Nous avons souhaité que les élèves de $1^{\prime}$ enseignement secondaire utilisent des problématiques liées à des domaines du réel pour valoriser leurs projets (Raynal et al., 2008, p.62).

L'utilisation de l'outil numérique dans un projet pédagogique interdisciplinaire pose également, comme le relève le rapport Jules Ferry 3.0 (Pène, 2014), d'autres questions sur les différences sociétales (Davidenkoof, 2014) telles que celle de l'apprentissage dans le milieu scolaire avec l'outil numérique. D'après Jacques-François Marchandise (entretien fait par Régis Guyon 2016), certaines populations ont une bonne connaissance de l'informatique mais ne savent pourtant pas communiquer et créer du lien social (relations, réseau personnel et professionnel). Dans certains cas, l'outil informatique joue même le rôle de filtre ; c'est le cas pour les autistes, par exemple, qui communiquent à travers l'outil informatique. L'auteur insiste aussi sur la notion d'apprentissage des connaissances du numérique comme des " méta-compétences » et sur l'importance de pousser les jeunes à réaliser des démarches de projet dans ce domaine. 
Notre proposition de recherche s'insère résolument dans cette perspective en intégrant du lien social dans le processus d'acquisition et de maîtrise des compétences.

\section{Mise en place du projet}

Deux réunions avec deux enseignants en STI2D (Sciences et technologies de l'industrie et du développement durable) du lycée Gaspard Monge, Jérôme Grizon et Antoine Costanza, ont été organisées ; notre travail autour du projet Tala Sound fut accueilli chaleureusement par l'équipe éducative, qui l'a même intégrée en tant que prochain sujet d'épreuve au baccalauréat technologique. Quand nous nous sommes présentés auprès de ces enseignants, nous leur avons simplement soumis un besoin technique, sans imposer de forme ou d'idée du dispositif, car la composante "design" fait partie intégrante du programme STI2D. C'est une partie de coconstruction des compétences des élèves, spécifiquement intégrée dans les ressources pour le cycle de la classe de terminale ${ }^{10}$ de 2011 (prolongé jusqu'à 2021) de l'Education nationale. Ces spécifications pédagogiques intègrent une importante partie consacrée au design, "Architecture et design de produits», considérés comme «facteur d'humanisation innovante », tout comme pour les milieux médical (p. 98) et sonore (p. 59) (DGESCO, 2011).

Tala Sound est donc maintenant officiellement une épreuve de type Projet en enseignement spécifique à la spécialité (BO du 23 octobre 2014 : baccalauréat général série scientifique et technologique série STI2D). Pour favoriser la collaboration entre les différentes parties prenantes dans la réalisation du projet et renforcer la partie sociale de notre méthodologie de design, une présentation du projet Tala Sound a d'ailleurs été faite directement auprès des élèves. L'objectif était donc de mettre en place une méthodologie innovante en reliant autour d'un projet social l'enseignement secondaire et supérieur, c'est-à-dire de partir du bas vers le haut : "In any case, the relative novelty of "project-oriented" pedagogy is, in design schools in higher education where knowledge is traditionally transmitted from top to bottom, its supposed value as a learning model. » (Bertilorenzi et al., 2017, p.63).

Nous sommes conscients du coût en temps de cette approche inattendue et du risque sur le résultat final du projet. Mais la finalisation du dispositif de notre projet de recherche n'est pas notre seul objectif ; un autre enjeu important, avec le design social, consiste à créer des liens entre les différents pôles en jeu (voir la figure 1). "Dans toute activité humaine quotidienne (travail, école, vacances, alimentation, santé, loisirs, parentalité, culture, hygiène, etc.) convergent et s'entrechoquent des dimensions et des valeurs diverses et interdépendantes (économiques, sociétales, psychologiques, technologiques, historiques, sémiotiques, etc.) 》 (Findeli, 2015, p.54). Ainsi, les élèves auront à interagir et à gérer des relations avec différents environnements ; "The human-environment interaction should be understood as the interaction between the human social system and the rest of the ecosystem » (Marten, 2001, p. 34). L'acquisition de compétences en ce domaine constitue, résolument, un point important de notre projet.

De plus, l'intervention de ces élèves peut faire émerger des lignes de recherche non anticipées. Méthodologiquement, l'ensemble de ces développements fait ainsi indirectement référence à la notion de sérendipité, telle qu'elle est en particulier évoquée dans la théorie C-K (Hatchuel et al., 2008) : ici, elle est mise en action à partir d'observations de musique méditative, tout en utilisant un prisme d'analyse nouveau fondé sur ce que l'on ne connaît pas d'emblée (ici, le

\footnotetext{
${ }^{10} \mathrm{https}$ //eduscol.education.fr/sti/sites/eduscol.education.fr.sti/files/textes/formations-bac-technologique-bactechnologique-sti2d/51-ressources-sti2d.pdf
} 
milieu médical) et l'irruption de facteurs sociaux conjoncturels, le tout pour faire émerger une solution hybride (Dautrey et Quinz, 2014) non postulée a priori.

Figure 2 - Cartographie de l'innovation sociale par projet interdisciplinaire.

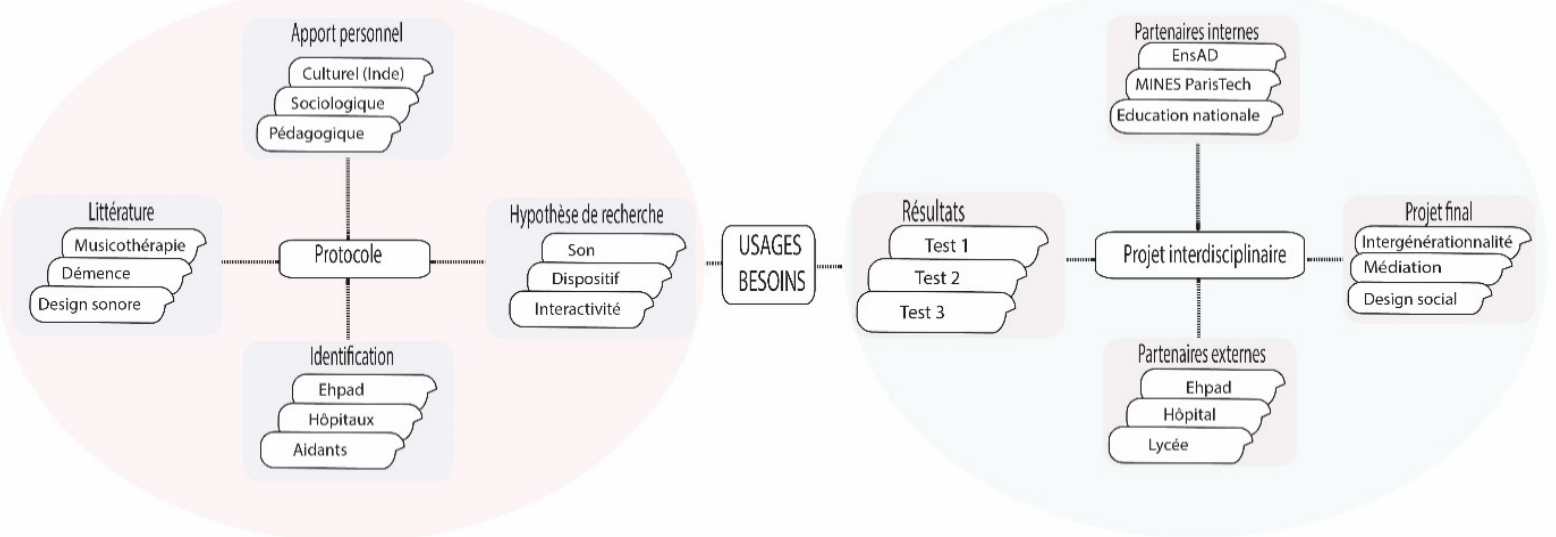

\section{Discussion}

Pour résumer, nous avons proposé ici une analyse du déroulé temporel d'un cas d'étude de recherche par le design dans le cadre du projet de design sonore médical Tala Sound, intégrant un futur objet sonore utilisant des rythmes carnatiques pour - telle est l'hypothèse à valider apaiser les patients Alzheimer. Nous avons vu que plusieurs approches existantes de recherche ont pu être mobilisées. La figure 2 récapitule les étapes clés de ce processus de recherche de manière schématique. Comme il serait vain d'espérer un simple parcours séquentiel, de la gauche vers la droite dans le schéma, lors de la réalisation d'un projet de design de ce type, les transitions entre les différentes étapes sont indiquées par des flèches bidirectionnelles, réversibles, ce qui permet de réévaluer à chaque étape les décisions précédentes. Ainsi, à titre d'illustration, dans le cas de Tala Sound, nous avons itéré le processus de test afin de trouver le temps d'exposition sonore le plus adapté à la population visée. Enfin, nous avons pris soin d'utiliser sur la figure 2 une représentation en profondeur pour illustrer les aspects pluridisciplinaires de notre méthodologie ; ceux-ci doivent, bien évidemment, être adaptés à chaque projet qui souhaiterait suivre nos préconisations.

En ce qui concerne le projet Tala Sound, une fois mis en place un contexte (ici, musique indienne et santé), nous avons premièrement utilisé une approche inductive en visitant les organismes pertinents, pour extraire une hypothèse qui se devait d'être en phase avec les protocoles de recherche dans le domaine de la santé. Deuxièmement, nous avons abordé la question des méthodes d'évaluation de l'efficacité d'un design, en mettant ici en œuvre deux sondages musicaux, lors d'un concert de musique indienne et au sein d'un centre d'accueil pour personnes âgées. Troisièmement, nous avons utilisé une approche par "le faire", par exemple lors de la composition de divers rythmes sonores, fonction des résultats des deux études. Enfin, notre démarche nous a conduits à faire l'hypothèse qu'il serait positif pour toutes les parties prenantes du projet d'envisager le processus global de développement sous l'angle du design social. Nous avons ainsi présenté notre projet à un établissement public du secondaire dans la banlieue parisienne, en créant du lien social entre les différents partenaires, tout comme le futur 
objet sonore, la Tala Box, créera, nous l'espérons, du lien entre patients et soignants (Valenti et al., 2015).

D’une manière plus générale, notre approche méthodologique de projet peut donc s'analyser selon deux angles : un versant relevant du (co-)design social, avec l'intervention de partenaires du monde de l'éducation et des technologies pour ce qui concerne la conception et la réalisation du dispositif ; un côté plus lié à l'interdisciplinarité, par exemple en ce qui concerne la perception (pour le choix des matériaux du dispositif ou des sons) ou la musicothérapie. Dans les deux cas, notre projet déplace les lignes, conduisant à des redéfinitions et/ou extensions de domaines d'étude préexistants. Ainsi, nous avons créé des ponts, rares jusqu'ici, entre le monde de l'enseignement en milieu scolaire et celui de la recherche. Sur le plan de la musicothérapie, notre positionnement sur la musique indienne, peu abordée dans le monde de la musicothérapie occidentale, qui se fonde essentiellement sur la création de réminiscences par le contact à des musiques familières aux patients, va peut-être (des expériences plus avancées pour éclaircir ce point sont prévues) conduire à redéfinir les outils de cette discipline. On le voit, notre approche interdisciplinaire et sociale du design sonore pour la santé ouvre des perspectives inédites, qui nous restent, bien évidemment, à consolider.

\section{Conclusion}

Élaborer un projet interdisciplinaire et social comme Tala Sound, autour d'un dispositif sonore pour patients atteints d'Alzheimer, demande la mise en place de relations de confiance entre des partenaires de disciplines parfois très éloignées. Nous étions conscients que nous ne pouvions pas intervenir dans un espace médical sans avoir réalisé, au préalable, un audit de l'existant. Mais cela ne pouvait suffire pour guider notre processus créatif autour de Tala Sound ; nous avons dû tout à la fois composer des musiques dédiées, les faire écouter, modifier certains items de notre cahier des charges, réfléchir au futur dispositif sonore Tala Box et trouver des partenaires motivés et permettant d'enrichir l'ensemble de la démarche. Cette approche, à la fois au niveau du fond et de la forme, par "le faire", la pratique et la théorie, explore tous les champs de connaissances des différents spécialistes.

Comme la recherche présentée est naturellement toujours en cours, la principale étape à venir consistera à tester en milieu hospitalier et/ou de soins pour personnes âgées l'objet Tala Box. Des évaluations à la fois quantitatives et qualitatives devront être effectuées afin d'estimer son impact sur les conditions de vie des patients et soignants. La démarche interdisciplinaire qui a été la nôtre semble tout à la fois nécessiter et favoriser le lien social entre les différents partenaires ; un projet tel que Tala Sound peut même, on l'a vu, faire sens au-delà de son domaine d'utilité initialement prévu et devenir un vrai outil pédagogique. Notre proposition est que ce type de démarche méthodologique innovante, interdisciplinaire et sociale, devrait pouvoir être utilisée dans d'autres projets en design. 


\section{Bibliographie}

ARS (2018), Environnement sonore des nouveau-nées prématurés dans des services hospitaliers franciliens, Rapport de Agence Régionale de Sante Île-De-France.

AKRICH, M. (1998), Les utilisateurs, acteurs de l'innovation, Revue éducation permanente, 134, 79-90.

AMBrosi, A., PEUGEOT, V., PIMIENTA, D. (2005), Enjeux de mots: regards multiculturels sur les sociétés de l'information, $\mathrm{C} \& \mathrm{~F}$ Éditions.

AFITEP, (2010) Dictionnaire de management de projet, Association francophone de management de projet AFNOR.

BEAUVAIS, C., JENSON J. (2002), Social Cohesion: Updating the State of the Research, Discussion Paper, Discussion paper No. F|22, Canadian Policy Research Networks.

BERTILORENZI, M., RUANO-BORBALAN, J.C., LE COQ, M. (2017), Between Innovation and Tradition: French Design Schools, their Historical Roots and their Innovation System, Journal of Innovation Economics Management, 1(22), 57-78.

BEST, K. (2009), Le design management, stratégie, méthode et mise en ouvre, Edition Pyramyd NICV.

BLAIS, M., MARTINEAU, S. (2006), L'analyse inductive générale : description d'une démarche visant à donner un sens à des données brutes, Recherches qualitatives, 26(2), 1-18.

BOBRIE, F. (2017), Sonifier le marché. Perspectives sémiotiques pour un design sonore marchand, Communication langages, 193(3), 89-100.

BONTEMS, V. (2014), What does Innovation Stand for? Review of a Watchword in Research Policies, Journal of Innovation Economics Management, 15(3), 39-57.

BOUILLON, D., GUILLERME, A., MILLE, M., PIERNAS, G. (2017), Gestes techniques, techniques du geste, Villeneuve d'Ascq, Presses Universitaire du Septentrion.

BOURGEAU, A. (2010), Le tabla et le tala dans les pratiques musicales européennes et nordaméricaines des années 1960 à nos jours, Volume !. La revue des musiques populaires, 7(2), 161-183.

BRAHMI, R., OUAMERI, S., TOUMI, J., BOULARDIN, E., TRIVALLE, C. (2019), Intérêt de la musicothérapie dans les troubles du comportement chez des patients déments hospitalisés, NPG Neurologie-Psychiatrie-Géroatrie, 19(111), 162-168.

CARDON, D. (2006), La trajectoire des innovations ascendantes : inventivité, coproduction et collectifs Internet. Innovations, usages, réseaux.

CHELKOFF, G. (1993), Du confort acoustique au confort sonore : Evolutions des pratiques et de l'architecture $d u$ logement. CRESSON Centre de recherche sur l'espace sonore et l'environnement urbain. UNRA CNRS.

CHOAIN, L. (2014), Permettre plutôt que transmettre : Face à la diversité des générations, comment les organisations peuvent-elles favoriser la coopération intergénérationnelle ? Question de management, 6, 125-149.

CÔTÉ, V., BÉLANGER, L., GAGNON, C. (2017), Le design au service de l'expérience patient Sciences du design, (2), 54-64.

CROWE, D., ZARCO, W., BARTOLO, R., MERCHANT, H. (2014), Dynamic Representation of the Temporal and Sequential Structure of Rhythmic Movements in the Primate Medial Premotor Cortex, Journal of Neuroscience, 34(36), 11972-11983.

CRUM, R.M., ANTHONY, J.C., BASSETT, S.S., FOLSTEIN, M.F. (1993), Population-based Norms for the Mini-mental State Examination by Age and Educational Level, JAMA, 269, 2386-2391. 
DAUTREY, J., QUINZ, E. (2014), Strange Design, Du design des objets au design des comportements, Edition IT.

DAVIDENKOFF, E. (2014), Le tsunami numérique, Stock.

DEBOS, F., LACROIX, C., CYRULNIK, N., RASSE, P., TROUSSE, B. (2017), Démarche de co-conception citoyenne dans les processus d'innovation par les usages : le cas du projet Ecofamilies, Information, Organisation et Connaissance.

DGESCO (2011), Enseignements technologiques transversaux et enseignements spécifiques (série STI2D), Eduscol, Ministère éducation nationale jeunesse vies associative.

DUBUISSON, S. HENNION A. (1996), Le Design : l'objet dans l'usage, Paris, Presses des Mines.

DONNAT, O. (2009), Les pratiques culturelles des Français à l'ère numérique : Éléments de synthèse 1997-2008, Culture études, 5(5), 1-12.

FAGNY, M. (2000), L'impact de la technique du «snoezelen» sur les comportements indiquant l'apaisement chez des adultes autistes, Revue Francophone de la déficience intellectuelle, vol.11, numéro 2, 105-115.

FERRARO, S. (2013), Art-thérapie et psychanalyse : vers une articulation théorique et clinique, Enfances \& Psy, 2(59), 174-181.

FINDELI, A. (2015), La recherche-projet en design et la question de la question de recherche : Essai de clarification conceptuelle, Science du design, (1), 45-57.

FUJIOKA, T., TRAINOR L.J., LARGE, E.W., ROSS, B. (2012), Internalized Timing of Isochronous Sounds Is Represented in Neuromagnetic Beta Oscillations, The Journal of Neuroscience, 32(5), 1791-1802.

GAGNON, C., COTE, V. (2016), Design public et innovation sociale : apprendre de la recherche appliquée, Actes du DRS 2016, Conférence du 50e anniversaire de la Design Research Society, Brighton, Royaume-Uni.

GAGNON, C. (1986), Compte-rendu de Jacques HAINARD et Roland KAEHR (éds) : Objets prétextes, objets manipulés, Musée d'ethnographie, Neuchâtel, Anthropologie et sociétés, 10(1), 233-234.

GENTES, A. (2017), The In-Discipline of Design: Bridging the Gap Between Humanities and Engineering, Gewerbestrasse, Springer.

GUETIN, S., PORTET, F., PICOT, M.C., DEFEZ, C., POSE, C., BLAYAC, J.P., TOUCHON, J. (2009), Intérêts de la musicothérapie sur l'anxiété, la dépression des patients atteints de la maladie d'Alzheimer et sur la charge ressentie par l'accompagnant principal (étude de faisabilité), L'Encéphale, 35(1), 57-65.

GUILLOUX, G., LE BCEUF, J. (2017), Design et territoires de pratiques en santé : enjeux pour la recherche et la formation, Sciences du design, (2), 26-39.

GUYON, R. (2016), Entretien avec Jacques-François Marchandise : Notre capacité à choisir, à savoir apprendre à l'ère numérique, Diversité, 185(3).

HAMEL, J. (1995), L'interdisciplinarité. Fiction de la recherche scientifique et réalité de sa gestion contemporaine, L'Homme et la société, 116(2), 59-71.

HATCHUEL, A., LE MASSON, P., WEIL, B. (2008), Studying Creative Design: The Contribution of C-K Theory, Conference Studying design creativity: Design Science, Computer Science. Cognitive Science and Neuroscience Approaches.

HEGDE, S. (2017), Sciences cognitives et traditions d'enseignement oral de la musique classique indienne, Revue internationale d'éducation de Sèvres, (75), 75-85.

HUEBER, T. (2009), Reconstitution de la parole par imagerie ultrasonore et vidéo de l'appareil vocal : vers une communication parlée silencieuse, Thèse de Doctorat, Université Pierre et Marie Curie Paris VI.

HUMMEL, C., HUGENTOBLER, V. (2007), La construction sociale du « problème » intergénérationnel, Gérontologie et société, 30(4), 71-84. 
KALYANI, B.G., $\quad$ VENKATASUBRAMANIAN, G., $\quad$ ARASAPPA, R., $\quad$ RAO, N.P., KALMADY, S.V., BEHERE, R.V., RAO, H., VASUDEV, M.K., GANGADHAR, B.N. (2011), Neurohemodynamic Correlates of 'OM' Chanting: A Pilot Functional Magnetic Resonance Imaging Study, International Journal of Yoga, 4(1), 3-6.

KUHN, P. (2012), Etude de la sensibilité auditive du nouveau-né grand prématuré aux stimulations sonores issues de son environnement, Thèse de Doctorat en Neurosciences, Université de Strasbourg.

LARROQUE, M. (2013), Les procédés de relaxation hier et aujourd'hui, L'information psychiatrique, 89(9), 751-758.

LAUQUIN, M., MINVIEILlE, N. (2015), Are You Design? du design thinking au design doing, Editions Pearson.

LEROUX, M., BARDYN, J-L. (2003), A l'écoute de l'hôpital, Rapport de recherche du Centre de Recherche sur l'Espace Sonore et l'Environnement urbain.

MARTEN, G. (2001), Human Ecology - Basic Concepts for Sustainable Development, London \& Sterling, Earthscan Publications.

MARTIN, B. (2012), Analyse de structures répétitives dans les séquences musicales, Thèse de Doctorat, Université Sciences et Technologies - Bordeaux I.

MISDARIIS, N. (2018), Sciences du Design Sonore - Approche intégrée du design sonore au sein de la recherche en design, HDR, Université de Technologie de Compiègne (UTC).

MORILLON, B., BAILLET, S. (2017), Motor Origin of Temporal Predictions in Auditory Attention, Proceedings of the National Academy of Sciences, 114(42), E8913-E8921

MOUSSARD, A. (2012), L'utilisation de la musique comme support de nouveaux apprentissages dans le vieillissement normal et la maladie d'Alzheimer, Thèse de Doctorat de Psychologie, Université de Bourgogne.

MOUSSARD, A., ROCHETTE F., BIGAND. E. (2012), La musique comme outil de stimulation cognitive, L'Année psychologique, vol. 112, no. 3, 2012, pp. 499-542.

MOYLE, W., COOKE, M., BEATTIE, E., JONES, C., KLEIN, B., COOK, G., GRAY, C. (2013), Exploring the Effect of Companion Robots on Emotional Expression in Older Adults with Dementia: A Pilot Randomized Controlled Trial, Journal of Gerontological Nursing, 39(5), 46-53.

PENE, S., ABITEBOUL, S., BALAGUE, C., BLECHER, L., BLOCH-PUJO, N., BRIAND, M., GARCIA, C., JUTAND, F., KAPLAN D., LUCIANI-BOYER, P., PEUGEOT, V., STIEGLER, B., VALEE, B. (2014), Jules Ferry 3.0 : bâtir une école créative et juste dans un monde numérique, Rapport du Conseil National du Numérique.

PLATON, (2016), La République, Flammarion.

RAYNAL, S., LE MEHAUTE, A., ANGOUlVANT, F., FERGUSON, L. (2008), De la pédagogie de projet à la conception de projet : Construction d'un bateau pour la compétition transatlantique, La revue des sciences de gestion : Direction et gestion, 43(231-232), 53-63.

THOREZ, D., NOËL, J., DE MONTGOLFIER, S., LE DASTUMER, B. (2009), Le libre choix du patient dément en institution, Gérontologie et société, 32(4), 131-146.

VALENTI SOLER, M., AGÜERA-ORTIZ, L., OLAZARAN RODRIGUEZ, J., MENDOZA REBOLLEDO, C., PEREZ MUÑOZ, A., RODRIGUEZ PEREZ, I., OSA RUIZ, E., BARRIOS SANCHEZ, A., HERRERO CANO, V., CARRASCO CHILLON, L., FELIPE RUIZ, S., LOPEZ ALVAREZ, J., LEON SALAS, B., CAÑAS PLAZA J.M., MARTIN RICO, F., ABELLA DAGO, G., MARTINEZ MARTIN, P. (2015), Social Robots in Advanced Dementia, Frontiers in Aging Neuroscience, 7, 133.

VIAL, S. (2015), Le Design : Que sais-je ? n 3991, Presses Universitaires de France. VRAI 
